\title{
FLOOD MAPPING USING INSAR COHERENCE MAP
}

\author{
Slim Selmi ${ }^{\mathrm{a}}$, Wajih Ben Abdallah ${ }^{\mathrm{a}}$, Riadh Abdelfattah ${ }^{\mathrm{a}, \mathrm{b}}$ \\ ${ }^{a}$ COSIM Lab, University of Carthage, Higher School Of Communications of Tunis \\ Route de Raoued KM 3.5, Cite El Ghazala Ariana 2083 - Tunisia, email:slim.selmi@ supcom.tn \\ ${ }^{\mathrm{b}}$ Département ITI, Télécom Bretagne, Institut de Télécom, Technopôle Brest-Iroise CS 83818 \\ 29238 Brest Cedex 3 - France, email:riadh.abdelfattah@supcom.rnu.tn
}

KEY WORDS: Flood detection, SAR Data, InSAR coherence map.

\begin{abstract}
:
Classic approaches for the detection of flooded areas are based on a static analysis of optical images and/or SAR data during and after the event. In this paper, we aim to extract the flooded zones by using the SAR image coupled with the InSAR coherence. A new formulation of the ratio approach for flood detection is given considering InSAR coherence. Our contribution is to take advantage from the coherence map provided using the InSAR pairs (one before and one after the event) to enhance the detection of flooded areas. We explore the fact that the coherence values during and after the flood are mainly differents on the flooded zones and we give a more suitable flood decision rule using this assumption. The proposed approach is tested and validated in the case of the flood taken place in 2005 in the region of Kef in Tunisia.
\end{abstract}

\section{INTRODUCTION}

Earth has experienced in recent years, several natural disasters that have an environment impact as well as various aspects of human life (Zhou et al., 2000). One of these remarkable events is floods. In Tunisia, the risk of floods affects about two out of three municipalities (of the Directorate General of Water Resources, 2005). It is therefore necessary to provide objective and comprehensive information about the area before, during and after this hydrological event(Zhou et al., 2000). To guide support effectively and to provide quantifiable estimates of affected infrastructure and land extent, remote sensing techniques are used to measure and monitor floods (Andreoli and Yesou, 2007). Indeed, remote sensing data integration allows calculation and rapid assessment of water levels, damages and flood risk by area (Zhou et al., 2000), (Andreoli and Yesou, 2007).

In this study, two main methods based on SAR (Synthetic Aperture Radar) data, for the extraction of flooded areas are studied and compared. We then propose an optimization of the more accurate one: the ratio approach (Andreoli and Yesou, 2007). The first method is developed by NEST (Next ESA, all rights reserved, n.d.) and it is based on the determination of a mask from the color composite images taken before and during the flood. Andreoli and Yesou developed an approach based on the texture filtering (Andreoli and Yesou, 2007) and the detection of the change through the bands ratio (Andreoli and Yesou, 2007). In order to overcome the problem of decorrelation due to the landslide caused by the flood, we used the InSAR coherence as an additional information for texture filtering. Thus, flood mapping using ETM+ optical data is considered as a "ground truth" (Van Leer and Galantowicz, n.d.) for the validation and comparison of these methods.

\section{FLOODS IN NORTHWESTERN OF TUNISIA}

We are interested in the region of Kef in the north-west of Tunisia Fig. 1. This region is knowing for having a semi-arid climate and it becomes inaccessible after floods. This will make very difficult the task of research looking for exploring the test site within field campaign after a flooding.

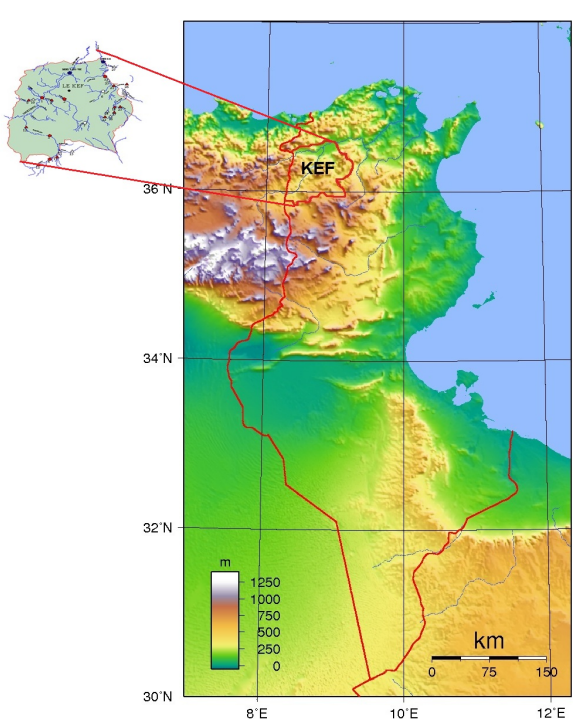

Figure 1: Location of the study area $\left(36^{\circ} 10^{\prime} 55^{\prime \prime} \mathrm{N}, 8^{\circ} 42^{\prime} 52^{\prime \prime} \mathrm{E}\right)$.

According to the Tunisian hydrological calendar, published by the Ministry of Agriculture and Water Resources (of the Directorate General of Water Resources, 2005), February is the month of greatest rainfall. Table 1 shows data on precipitation in 2005 . Floods in Kef is taken place between February $14^{t h}$ and $15^{\text {th } 2005}$. Researchers took more then 2 months (until April) in order to get on the test site. This mainly due to the landslides caused by the flooding.

In order to get a priori estimation of the flood mappping, we propose to explore different approaches based on SAR data. In this paper, flood mapping using optical data will serve as reference for the validation of results given from SAR approaches.

\section{FLOOD MAPPING USING OPTICAL DATA}

A classic way to detect the flooded areas is based on the analysis of the optical images. The main idea is to test different processing satellite data to extract thematic land such as principal 
Table 1: The amount of rain in 2005 in three rainfall stations in the region of Kef.

\begin{tabular}{llllllll}
\hline Station & Jan & Feb & Mar & Apr & May & Jun & Jul \\
\hline Mellegue & 41.0 & $\mathbf{6 5 . 0}$ & 34.8 & 53.0 & 8.0 & 23.6 & 4.8 \\
Zebida & 42.0 & $\mathbf{6 8 . 0}$ & 22.8 & 34.0 & 0.0 & 14.0 & 4.0 \\
K. Khasba & 42 & $\mathbf{6 3 . 0}$ & 27.0 & 40.0 & 6.0 & 77.0 & 47.0 \\
\hline
\end{tabular}

component analysis, the colored composition, multispectral classification and the band ratio $4 / 3$ (Roque et al., 2013). Because the Kef test site is mainly composed of soil, vegetation and water (Roque et al., 2013), we opted for the choice of the band ratio $4 / 3$ since it maximizes classes separability (of Environment and of Life Branch, 2006).

The use of Landsat ETM+ tool for images processing and analysis has identified the most relevant elements of the landscape and draw up land using maps (Fig. 2).

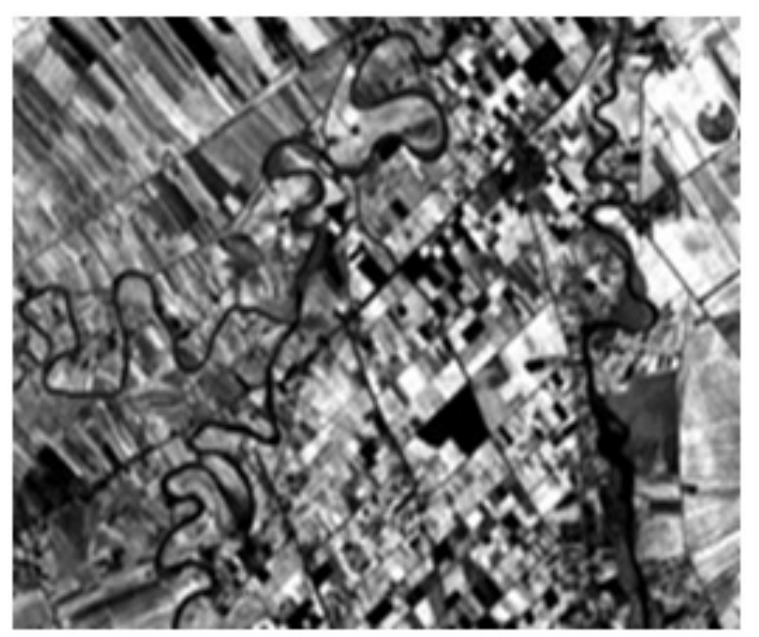

Figure 2: Optical image of river Mellegue taken by Landsat ETM+ satellite on Mai 2005 with band ratios 4/3.

The pixels having values below 1.76 denote the surfaces of water. Among water regions, some aren't related to flooding (overflow from the river due to the high flood waters and stagnant rain). By examining the optical images taken over the region of Kef in Tunisia on Marsh and May 2005, we can detect the flooded areas. The resulting map shows the areas that are affected during the crisis. This will save for us as a ground truth for validation of the developed approach. During the flood(in February) and immediately after the flood, optical data are not exploitable. Then, SAR data in such situation are more adapted for flood mapping.

\section{FLOOD MAPPING USING SAR DATA}

The radar data set used in this paper is provided by Envisat-2 satellite covering northwest of Tunisia and taken between February 2005 (during the flood) and May 2005 (after the flood).

\subsection{Methodology}

The methodology of radar data processing consists of three steps (Andreoli and Yesou, 2007)(Silveira and Heleno, 2009):

- Geo-location of radar images

- Radiometric processing

- Image interpretation and information extraction.
For our study, we used the Lee filter (Lee et al., 1998) to reduce speckle noise and NEST tool (Next ESA, all rights reserved, n.d.) to process image data and metadata. The radar sensors have a high sensitivity to the dielectric constant and the surface roughness of observed objects. If we assume that a free water surface has a very low roughness, we can consider it as specular reflector. Thus, water is distinguished with a very low image backscatter (Roque et al., 2013)(Silveira and Heleno, 2008).

4.1.1 Detection of flood using a mask To measure the extent of floods, the permanent water surfaces shouldn't be considered. Otherwise, we will get erroneous results. This implies that we need to hide some pixels and expose others. Before creating a mask, the RGB image will be used where the red band denotes the crisis image (February), the green band denotes the archive 1 and the blue band is or the archive 2 (Next ESA, all rights reserved, n.d.). From these images, we can see pixels associated with flooded areas colored in purple. Knowing these values, and based on histograms of images taken before and after the floods, thresholds could be defined. Therefore, the permanent water surfaces could be detected using these thresholds. Using these data, we can notice that the pixel values, in the case of the crisis image (14 February), are below thresholds (from 250 to 350) but in the case of the picture archive, the same pixels values are between 400 and 600 . So the flooded areas satisfy the condition of $N$ mask (Fig. 3):

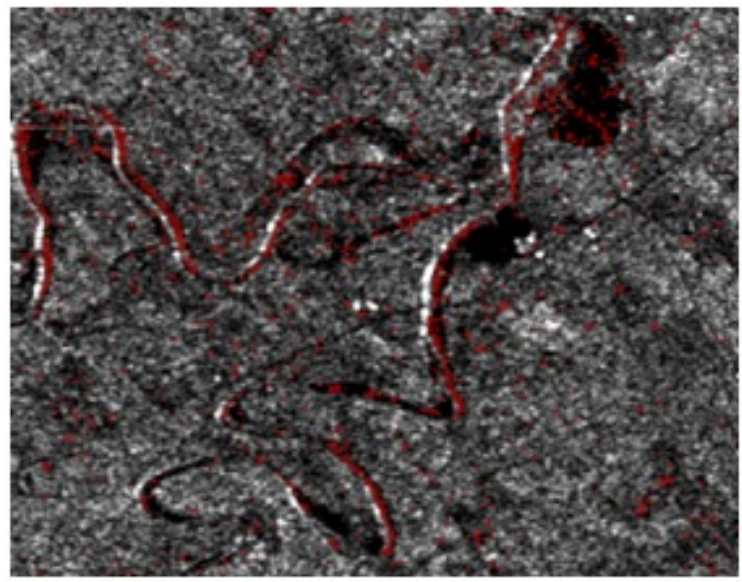

Figure 3: Flooded area (in red) around river Mellegue generated with Envisat satellite image on Mars 2005 using NEST method.

$$
N:\left\{\begin{array}{l}
I_{t}<350 \\
I_{t+1}>550
\end{array}\right.
$$

where $I_{t}$ and $I_{t+1}$ are the Envisat images taken during and after the flood event repectively.

4.1.2 Detection of flooding using the ratio method The changing detector chosen for this method is based on the improvement of the ratio that includes two levels of analysis (Roque et al., 2013), the macro-scale analysis based on a first ratio $r_{1}$ in order to highlight changes of large homogeneous areas and combined with a second ratio $r_{2}$ obtained from two raw images that highlight key changes, while keeping a thematic accuracy of the raw data (Roque et al., 2013)(Rignot and van Zyl, 1993).

$$
r_{1}=\frac{I_{1, t+1}}{I_{1, t}} \quad r_{2}=\frac{I_{2, t+1}}{I_{2, t}}
$$

where:

$I_{1, t}:$ Texturally filtered image at a time $\mathrm{t}$. 
$I_{1, t+1}:$ Texturally filtered image at a time $\mathrm{t}+1$.

$I_{2, t}$ :Original image at a time t.

$I_{2, t+1}$ :Original image at a time $\mathrm{t}+1$.

\section{PROPOSED APPROACH : FLOOD DETECTION USING RATIO AND CHOERENCE MAP}

This approach combined the ratio $r$ and consistency $\gamma$ of radar images $I_{t}$ and $I_{t+1}$ to determine any changes between areas and therefore the extraction of flooded zones. In fact, since $\gamma$ measured the similarity degree between these two images, any changes occured during the flood event could be detected if the value of $\gamma$ is small. In this proposed appraoch, our main contribution is to explore this assumption by using the coherence information between $I_{t}$ and $I_{t+1}$ to improve the flood detection. In our study, the sar images are acquired with the Envisat satellite, taken during and after the flood event.

The coherence image is simply the module of this complex coefficient $\gamma$ (Giordano et al., 2005) .

$$
\gamma=\frac{\sum_{j=1}^{N} I_{t}(\mathrm{~J}) I_{t+1}^{*}(\mathrm{~J})}{\sqrt{\sum_{j=1}^{N}\left|I_{t}(\mathrm{~J})\right|^{2} \sum_{j=1}^{N}\left|I_{t+1}(\mathrm{~J})\right|^{2}}}
$$

This is actually a picture of correlation between the two images $I_{t}$ and $I_{t+1}$. Indeed, if two pixels are completely decorrelated, the coefficient $|\gamma|$ tends to 0 . In the opposite scenario, coherence approaches $1 . N$ is the number of pixels whose analysis window is equal to 3 (Liu et al., 2001). We propose to mode the change detection coefficient as given by the following expression:

$$
C_{r}=\frac{r}{\gamma}=\frac{1}{\gamma} \frac{I_{t+1}}{I_{t}}
$$

There are three possible cases:

Case 1: If the area undergoes no change then $r$ and $\gamma$ are close to 1 so the values of $C_{r}$ is around 1 .

Case 2: If the area undergoes negative changes (flooded areas) then $\gamma$ tends to 0 and $r>>1$ so the values of $C_{r}$ should be significant.

Case 3: If the area undergoes positive changes (non flooded areas) then $\gamma$ tends to 0 and $r<<1$ so the values of $C_{r}$ are less significant.

Whites areas, that appears in the changes detection coefficient image $C_{r}$ (Fig. 4), are flooded. Thus, flooded areas satisfy the condition $C_{r}>s_{1}$ where $s_{1}$ is a threshold to be determined. Threshold was chosen to minimize MSE (mean square error) between image $C_{r}$ and optical image, considered as land truth (Fig. 5).

$$
M S E=\frac{1}{M N} \sum_{i}^{M} \sum_{j}^{N}\left(C_{r}(i, j)-I_{o p}(i, j)\right)^{2}
$$

where $I_{o p}$ represents flooded areas mapping extracted from optical data.

\section{EXPERIMENTAL RESULTS AND DISCUSSIONS}

Exploited satellite database includes two ETM+ multispectral scenes: Marsh $14^{t h}, 2005$ in the raw, and May $1^{\text {st }}, 2005$ during the off

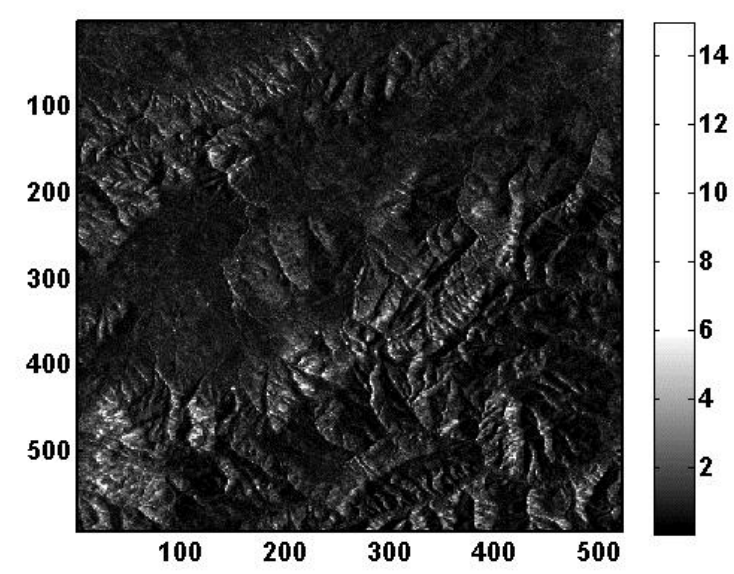

Figure 4: Change detection result using $C_{r}$ in the case of Envisat images.

flooding. We have calculated the $M S E_{1}$ between flooding images determinate from optical data (Fig. 6-(a)), considered as a reference image, and change detection image. The relationship between the threshold values and the obtained MSEs is shown in Fig. 5. $M S E_{1}$ decrease when the threshold increases. Starting from a particular threshold, we obtain $M S E_{1}=0.15$ and $M S E_{2}=0.18$ using mask method.

$$
\begin{aligned}
& M S E_{1}=\frac{1}{M N} \sum_{i}^{M} \sum_{j}^{N}\left(C_{s}(i, j)-I_{o p}(i, j)\right)^{2} \\
& M S E_{2}=\frac{1}{M N} \sum_{i}^{M} \sum_{j}^{N}\left(M(i, j)-I_{o p}(i, j)\right)^{2}
\end{aligned}
$$

Where:

$C_{s}$ : thresholded image of $C_{r}$.

$M$ : Flooding image using the mask.

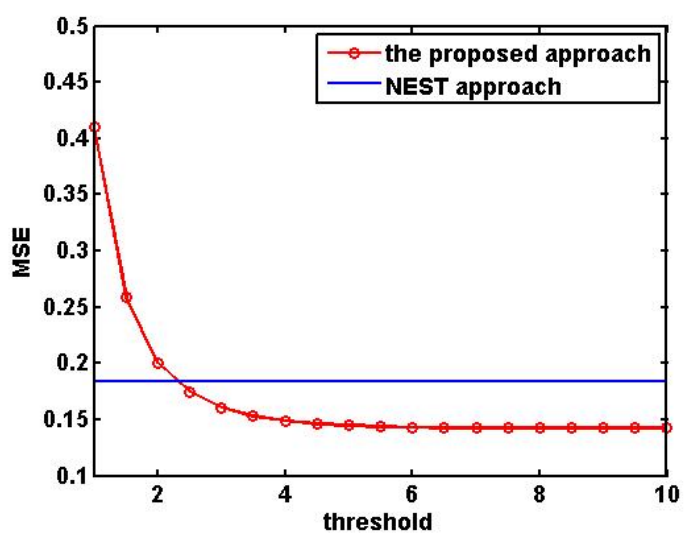

Figure 5: Relationship between the threshold values and the MSE of the NEST and the proposed approaches.

Fig. 6-(a) shows the flooded zones in area 1 of the Kef test site in 2005 extracted using ETM+ optical data. Since each pixel of the ETM+ image is $30 \times 30$ meters and the number of flooded pixel is 42104, the surface of the flooded area is calculated as following:

$$
S_{o p}=42104 \times 30 \times 30=37.89 \mathrm{~km}^{2}
$$


Thus, flooded zones represent about $14.22 \%$ of the total land area. Moreover, the spatial resolution of SAR images is $12.5 \times$ 12.5 meters. The flooding surfaces $S_{1}$ and $S_{2}$ obtained from the mask method and from the method of change detection area respectively are:

$$
S_{1}=30.65 \mathrm{~km}^{2}, S_{2}=40.74 \mathrm{~km}^{2}
$$

This means that the flooded zone covers $11.5 \%$ of the total land area for the method of mask and $15.28 \%$ for the method of change detection.

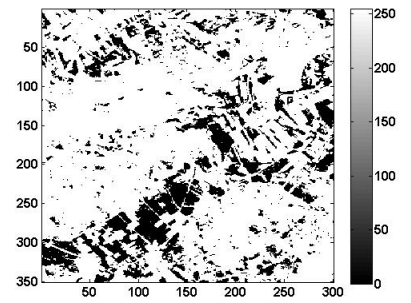

(a)

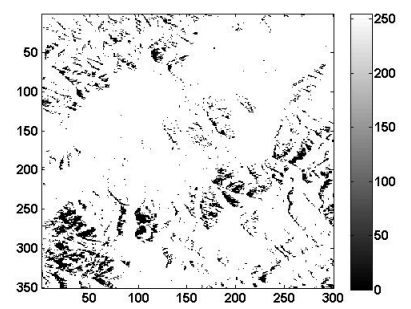

(c)

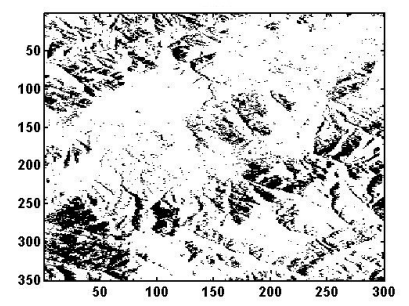

(e)

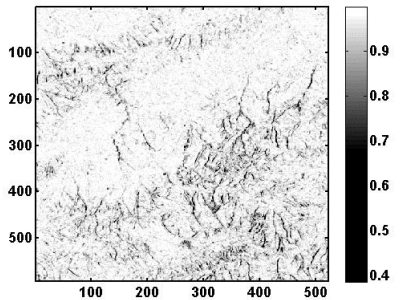

(b)

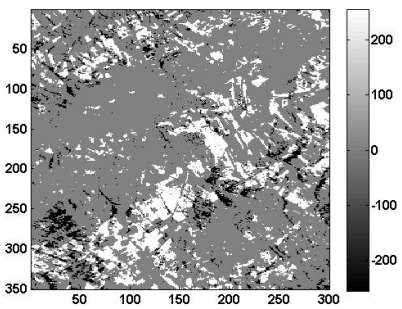

(d)

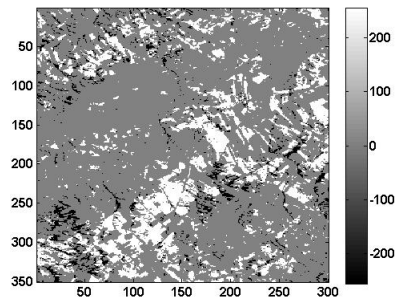

(f)
Figure 6: Flooded areas in black : (a) optical method, (b) coherence map, (c) mask method, (d) the difference image $(a)-(c)$, (e) the proposed approach and the difference image $(a)-(e)$.

\section{CONCLUSION}

The results presented in this paper demonstrate the relevance of the combination of satellite imagery (optical and radar) for mapping flooded areas. Indeed, processing and analysis of a multitemporal series of optical data, ETM+, and SAR data of the study area, acquired in different seasons, have identified a set of elements that can inform land uses in the area: vegetation units, soil and water. Furthermore, the multitemporal comparison between images was used to measure the surfaces of flooded areas around the river Mellegue and the impact of flooding on other region of the North-west of Tunisia. In this study, the ratio $4 / 3$ of optical bands allows the classification of land occupation. Furthermore, we have combined the ratio of SAR bands and the coherence in order to extract flooded areas more precisely.

As future work, we can use the elevation information of the soil, provided from the Digital Elevation Model (DEM) of the area of interest, to prevent the flooded zones with low elevation.

\section{REFERENCES}

Andreoli, R. and Yesou, H., 2007. Large scale change detection techniques dedicated to flood monitoring using envisat wide swath mode data. Geoscience and Remote Sensing Symposium, 2007. IGARSS 2007. IEEE International pp. 2382-2385.

Giordano, F., Goccia, M. and Dellepiane, S., 2005. Segmentation of coherence maps for flood damage assessment. In: Image Processing, 2005. ICIP 2005. IEEE International Conference on, Vol. 2, IEEE, pp. II-233.

Lee, J.-S., Papathanassiou, K. P., Ainsworth, T. L., Grunes, M. R. and Reigber, A., 1998. A new technique for noise filtering of sar interferometric phase images. Geoscience and Remote Sensing, IEEE Transactions on 36(5), pp. 1456-1465.

Liu, J. G., Black, A., Lee, H., Hanaizumi, H. and Moore, J. M., 2001. Land surface change detection in a desert area in algeria using multi-temporal ers sar coherence images. International Journal of Remote Sensing 22(13), pp. 2463-2477.

Next ESA, all rights reserved, n.d. http://nest.array.ca/.

of Environment, T. D. G. and of Life Branch, Q., 2006. Regional action program for fighting against desertification in the region of KEF. Ministry of Equipment, Land-Use Planning and Sustainable Development.

of the Directorate General of Water Resources, P., 2005. Annuaire hydrologique de tunisie 2004-2005.

Rignot, E. J. and van Zyl, J. J., 1993. Change detection techniques for ers-1 sar data. Geoscience and Remote Sensing, IEEE Transactions on 31(4), pp. 896-906.

Roque, D., Afonso, N., Fonseca, A. and Heleno, S., 2013. Building a database of flood extension maps using satellite imagery. Proccedings of the ESA Living Planet Symposium, Edinburgh September 9.

Silveira, M. and Heleno, S., 2008. Water/land segmentation in sar images using level sets. In: Image Processing, 2008. ICIP 2008. 15th IEEE International Conference on, IEEE, pp. 1896-1899.

Silveira, M. and Heleno, S., 2009. Classification of water regions in sar images using level sets and non-parametric density estimation. In: Image Processing (ICIP), 2009 16th IEEE International Conference on, IEEE, pp. 1685-1688.

Van Leer, K. W. and Galantowicz, J., n.d. Comparisons of flood affected area derived from modis and landsat imagery. pp. 1-8.

Zhou, G., Luo, J., Yang, C., Li, B. and Wang, S., 2000. Flood monitoring using multi-temporal avhrr and radarsat imagery. Photogrammetric Engineering and Remote Sensing 66(5), pp. 633-638. 